\title{
In-situ surface functionalization of superparamagnetic reduced graphene oxide - Fe3O4 nanocomposite via Ganoderma lucidum extract for targeted cancer therapy application
}

\begin{abstract}
A superparamagnetic graphene-based magnetite nanocomposite (rGO-Fe3O4) was synthesized via a simple in-situ chemical approach. This rGO-Fe3O4 nanocomposite can be used as a drug carrier that is able to be guided by external magnetic fields to the specific site of interest for targeted drug delivery application to treat cancer. Ganoderma lucidum extract (GL) was employed, which successfully stabilized the $\mathrm{rGO}-\mathrm{Fe} 3 \mathrm{O} 4$ via hydrogen bonding and resulted in enhancement of water dispersibility and stability of the prepared nanocomposite, while Pluronic F-127 (PF) was introduced to reduce the overall cytotoxicity. The presence of both GL and PF on the surface of nanocomposite was successfully validated by cyclic voltammetry (CV). Quercetin (Que), a naturally-available polyphenolic flavonoid with anti-cancer properties was utilized to study the potential of rGO-Fe3O4-GL-PF for controlled drug delivery application. The loading capacity of Que on rGO-Fe3O4-GL-PF was determined to be $11 \mathrm{wt} \%$ through UV-visible spectroscopy. The Que was loaded on rGO plane via $\pi-\pi$ stacking and hydrophobic interaction, which was validated through $\mathrm{CV}$. Furthermore, the in-vitro cytotoxicity of the synthesized nanocomposite showed obvious cytotoxicity toward A549 cells due to the anti-cancer properties of GL which has high potential to be developed into a targeted drug delivery carrier for cancer therapeutics.
\end{abstract}

Keyword: Superparamagnetic rGO-Fe3O4 nanocomposite; Ganoderma lucidum; Drug carrier; Quercetin; Cancer therapy 